\title{
Situación actual del sector energético en España
}

\author{
Carlos J. Pardo Abad (Doctor en Geografía)
}

\section{RESUMEN SUMMARY}

La energía es un sector clave de la economia española y está presente en todos los procesos productivos. El artículo analiza al sector en los últimos años con datos procedentes de varios organismos públicos, referidos tanto al balance energético general como a los sectores energéticos, las energías renovables, la política energética y las relaciones entre energía $y$ medio ambiente. Características básicas del sector en nuestro país son el incremento del consumo de energía, la progresiva liberalización y las grandes inversiones en energías renovables, sobre todo la eólica.
Energy is a key sector of the Spanish economy and it is in all productive processes. This article analyses the sector in the last few years with data from some public agencies, referred to the power balance as well as to nuclear power, coal, oil, natural gas, renewable energies, power policy and the relationship between energy and environment. The main eatures of energy in Spain are the increase of consumption, the growing liberation and the big investments in renewable energies, above all the eolic one.

\section{INTRODUCCIÓN}

En este artículo hacemos una presentación general del sector energético en España en los últimos años, atendiendo tanto a los diferentes sectores como a la política energética llevada a cabo en nuestro país. El 
consumo de energía se ha incrementado considerablemente y hoy en día alcanza cifras de país verdaderamente desarrollado. Pero no sólo el consumo, sino la actividad del sector energético en general. En algunas energías renovables, por ejemplo, España ha logrado convertirse en un país de referencia internacional.

Para ver la evolución de las principales variables, utilizaremos datos de 1997 y 1998 publicados por el Ministerio de Industria y Energía y por el Instituto para la Diversificación y Ahorro de la Energía en 1999. En este contexto, la demanda de energía ha aumentado en todos los usos finales, sobre todo en el ámbito de la industria y el transporte y como consecuencia de la evolución general de la economía.

La importancia de la energía es indiscutible para la práctica totalidad de los procesos productivos. España es un país que se caracteriza por la fuerte escasez de los recursos energéticos tradicionales: es el caso de los hidrocarburos, pero también del carbón por su deficiente calidad y carestía de explotación. Esto origina un gran déficit ante la débil producción nacional y la elevada demanda, con fuertes incrementos en periodos de expansión económica. La dependencia energética española en 1997 fue del $71 \%$, cifra muy elevada en comparación con otros países de nuestro entorno, y que obliga necesariamente a la búsqueda de alternativas y a la diversificación energética de cara a reducir una posición tan vulnerable ante posibles modificaciones del actual panorama internacional.

La línea principal de actuación en materia de política energética es la progresiva liberalización, reduciéndose cada vez más la intervención estatal, tal y como queda reflejado en la Ley 54/97 del Sector Eléctrico, que establece una nueva regulación del sector. Esta liberalización afecta también al petróleo y culmina en la Ley $34 / 98$ de Hidrocarburos. Esto se observa tanto en lo que se refiere a la distribución de carburantes y combustibles petrolíferos como a la privatización de compañías públicas del sector, como Repsol.

Las energias renovables han evolucionado en España muy favorablemente, con inversiones en este campo próximas a los 70.000 millones de pesetas anuales. Según datos proporcionados por la Oficina Estadística Europea, España ocupaba en 1997 la cuarta posición en producción total de energias renovables dentro de la Unión Europea, detrás de Francia, Suecia e Italia. En 1998, la aportación de las energias renovables al balance energético nacional fue del 6,3\%, destacando la eólica, que consiguió duplicar su aportación respecto al año anterior al alcanzar el 1,7\% del total, aunque a bastante distancia de la biomasa (54\%) y la hidroelec- 
tricidad $(44 \%)$, que siguen siendo las energías renovables de mayor importancia. A nivel más cualitativo que cuantitativo, también se han producido avances considerables en la energía solar, tanto térmica como fotovoltaica. Los resultados obtenidos en el campo de las renovables han sido, en conjunto, muy satisfactorios y pueden verse consolidados con la elaboración del Plan de Fomento de las Energías Renovables para el periodo 1999-2010.

En relación con la protección medioambiental, hay cada vez una mayor conciencia y un más amplio desarrollo normativo. Los acuerdos firmados a nivel internacional conllevan la realización de fuertes inversiones en el sector energético. Con ello se pretende tanto la reducción de las emisiones de todos los procesos productivos como la adaptación de los productos a las nuevas especificaciones. Esto, a su vez, incide en un incremento considerable en investigación y desarrollo tecnológico.

\section{BALANCE ENERGÉTICO}

La participación de cada fuente de energía en el consumo total de energía se puede observar a través de los denominados "balances energéticos", que van referidos a un espacio geográfico concreto y a un periodo de tiempo determinado. En definitiva, lo que se establece en un balance es el porcentaje y el valor absoluto de cada energía en el consumo energético total. Debido a las diferencias en las características de las distintas fuentes y a la transformación que precisan para su consumo, vamos a distinguir, tanto en demanda como en producción, la energía final de la energía primaria. La energía primaria también se denomina fuentes de energía o recursos energéticos. Se obtiene directamente de la naturaleza y es previa a cualquier transformación técnica. Energía final o útil es aquélla que utiliza directamente el consumidor después del proceso de transformación de la energía primaria. La producción de energía útil supone siempre el consumo de una determinada cantidad de energía primaria. Este consumo se reduce a medida que el nivel tecnológico aplicado en la transformación es mayor. Las pérdidas por transformación oscilan entre un $40 \%$ y un $50 \%$, lo que implica que las cifras referidas a energia primaria y final nunca coincidan.

Como ya quedó expuesto en la introducción, la dependencia energética española es muy elevada. Aparte de su incidencia sobre la balanza de pagos, las posibles conmociones internacionales pueden poner en peligro el suministro desde el exterior y generar incertidumbres en torno a la 
evolución del precio de los productos energéticos. Esta circunstancia, sobre la que volveremos más adelante, es la primera característica de la estructura energética de nuestro país. De momento cabe apuntar que las compras energéticas en el exterior suponen cerca del $40 \%$ de la simportaciones totales de mercancías, en torno al $60 \%$ de todas las exportaciones españolas y casi superan los ingresos por turismo. El reciente aumento del precio del petróleo, la principal energía importada, está agravando estos parámetros macroeconómicos.

Antes de analizar la actual demanda y producción de energía primaria en España, vamos a comentar el panorama energético desde los años 50 hasta los 90. Dicho periodo, siguiendo a García Alonso e Iranzo Martín (1989), presenta tres momentos diferenciados: década de los 50, coincidiendo con los inicios de la industrialización; década de los 60 y primera mitad de los 70, de acelerado crecimiento económico; y desde 1975 hasta 1990, aproximadamente.

a) Las características del panorama energético de los 50 se define por un débil consumo, una reducida diversificación y una escasa dependencia del exterior. La economía era fundamentalmente agraria, lo que motivaba un menor consumo al ser un sector de reducida demanda energética. El carbón centraba el consumo en un $73,6 \%$; el resto se repartía entre la hidroelectricidad $(17,5 \%)$ y el petróleo $(8,9 \%)$. Esta estructura era consecuencia de una política autárquica que procuraba satisfacer el consumo interno con producción nacional. El autoabastecimiento se situaba en el $90,5 \%$.

A lo largo de la década se incrementó de forma considerable la demanda de energía; aunque no se incorpora ninguna fuente nueva, se altera la importancia de las ya citadas, disminuyendo el carbón en más de veinte puntos $(50,2 \%$ del total en 1960) y aumentando el petróleo y la electricidad de origen hidráulico. El autoabastecimiento se reduce y se coloca en el $70 \%$ en 1960 . Es ahora realmente cuando se inicia la brecha entre producción nacional y consumo interior y que se irá agrandando en las décadas siguientes.

b) Durante los años sesenta y primera mitad de los setenta, el panorama cambió profundamente como consecuencia de un fuerte crecimiento económico, siendo el sector industrial el motor principal. Los precios baratos del petróleo favorecieron las importaciones y la apertura a los mercados energéticos internacionales. El consumo interior de energía primaria se multiplicó por tres en catorce años. Otro rasgo importante fue la 
creciente dependencia del exterior, lo que supuso un grave inconveniente ante las crisis petrolíferas de los años setenta. La sustitución del carbón por el petróleo, iniciada en la década de 1950, se aceleró ahora y desde 1965 el petróleo se convirtió en la principal fuente de energía en España, lo que se relaciona evidentemente con la intensa modernización económica del país.

También cabe destacar entre 1960 y 1974 la aparición de dos fuentes nuevas en el balance energético nacional: la energía nuclear y el gas natural. En agosto de 1969 entra en funcionamiento la central de Zorita (la primera central nuclear española), en 1971 lo hizo la de Santa María de Garoña y en 1972 Vandellós I. En 1969 también se incorporó el gas natural, con bastante retraso respecto a otros paises europeos debido al alejamiento de los principales yacimientos continentales. Pese al reequipamiento hidroeléctrico, la energía hidráulica vio descender posiciones relativas en el balance energético global, al pasar de representar el $19,1 \%$ del consumo total en 1960 al $12 \%$ en 1974 .

c) A partir de 1975 es cuando se empiezan a sentir los efectos de la primera crisis petrolífera. La demanda interna de energía decrece respecto a años anteriores. La gran dependencia del petróleo generada en los años del desarrollismo explica el fuerte impacto de la citada crisis, a lo que hay que añadir el retraso en la toma de medidas de ajuste para mejorar la situación. La demanda interna de energía siguió aumentando hasta 1979, para iniciar a partir de entonces un descenso que habría de durar hasta 1983. Desde 1986, el crecimiento del consumo energético siguió creciendo.

Hasta 1980 no se producen cambios sustanciales en la composición por fuentes del consumo español de energía. A pesar de la crisis, la economía española demostró muy poca flexibilidad y capacidad de adaptación. Durante los años ochenta comienza el descenso de la exagerada participación del petróleo. Eso se compensó con un aumento del carbón, lo que supuso invertir una tendencia de sustitución del carbón por el petróleo. También es verdad que dicho aumento no estuvo provocado ni por la industria ni por los transportes, sino por su utilización masiva en las centrales termoeléctricas.

Desde 1980 a 1990 aumentó también la energía nuclear, y la hidráulica, debido a las irregularidades climáticas de la península, presenta fuertes oscilaciones anuales. El gas natural siguió con cifras de consumo reducidas hasta 1986, relanzándose progresivamente a partir de entonces y hasta el momento actual. 
El déficit energético aumentó desde 1974 a 1979, para reducirse luego hasta1986. El déficit petrolero se ha reducido debido a que se ha sustituido por otras fuentes en la obtención de electricidad. El consumo y la red de distribución del gas natural se extendieron considerablemente.

\subsection{Demanda de energía primaria}

El consumo de energía primaria en España en 1997 fue de 107.015 ktep $(\mathrm{ktep}=$ miles de toneladas equivalentes de petróleo), con un incremento de casi el $6 \%$ sobre el de 1996 . El $80 \%$ del consumo del carbón correspondió a las centrales eléctricas. El consumo de petróleo fue de $57.452 \mathrm{ktep}$, con un aumento del 3,6\% respecto al año anterior. El gas natural fue el que registró una tasa de variación más positiva dentro del conjunto, al pasar su consumo de 8.401 ktep en 1996 a 11.057 ktep en 1997, lo que representa un aumento del $31,6 \%$. Las energías renovables, sin incluir a la hidráulica, suponen dentro del balance el 3,4\% del total, con $3.678 \mathrm{ktep}$. La energía hidroeléctrica y la eléctrica de origen nuclear son las únicas que registran variaciones negativas, con un $-12,2 \%$ y un $-1,8 \%$, respectivamente.

Cuadro 1. Consumo de energía primaria. 1996-1997

\begin{tabular}{lrrrrr}
\hline Fuente & \multicolumn{2}{c}{1996} & \multicolumn{2}{c}{1997} & Variación \\
& \multicolumn{1}{c}{ Ktep } & \multicolumn{1}{c}{$\%$} & \multicolumn{1}{c}{ Ktep } & $\%$ & \multicolumn{1}{c}{$\%$} \\
\hline Carbón & 15.547 & 15,4 & 17.617 & 16,5 & 13,3 \\
Petróleo & 55.433 & 54,8 & 57.452 & 53,7 & 3,6 \\
Gas natural & 8.401 & 8,3 & 11.057 & 10,3 & 31,6 \\
Nuclear & 14.680 & 14,5 & 14.411 & 13,5 & $-1,8$ \\
Hidráulica & 3.488 & 3,4 & 3.064 & 2,9 & $-12,2$ \\
Energías renovables & 3.541 & 3,5 & 3.678 & 3,4 & 3,9 \\
Saldo eléctrico (imp.-exp.) & 91 & 0,1 & -264 & $-0,2$ & - \\
Total & 101.181 & 100 & 107.015 & 100 & 5,8 \\
\hline
\end{tabular}

Ktep $=$ miles de toneladas equivalentes de petróleo.

Fuente: Ministerio de Industria y Energía 


\subsection{Demanda de energía final}

Esta demanda no coincide con los datos de la energía primaria ya comentados porque se obtiene como resultado de restar los consumos en los sectores energéticos (consumos propios y todos aquellos derivados de la transformación) y las pérdidas dentro de la cadena energética como consecuencia del paso de energía primaria a energía final, y que son, en mayor o menor medida, inevitables.

Aclarado lo anterior, el consumo de energía final en España durante el año 1997 fue $77.313 \mathrm{ktep}$. Si comparamos la cifra con las $74.010 \mathrm{ktep}$ de 1996 obtenemos un incremento de un 4,5\%. Esta evolución coincide con una fase de expansión económica en la que ha tenido un papel muy destacado la actividad industrial.

De todas las energías, ha sido el gas natural el que ha crecido por encima de todas las demás $(12,1 \%$ respecto a 1996). El consumo final de esta fuente en 1997 fue de $8.212 \mathrm{ktep}$. El consumo de electricidad para usos finales en 1997 fue de 155.371 GWh, que equivalen a $13.364 \mathrm{ktep}$. El incremento respecto al mismo consumo del año anterior es del $4,1 \%$. Parecida fue la evolución de los productos petrolíferos, cuyo consumo final ascendió a $50.108 \mathrm{ktep}$. También en este apartado están las actividades industriales y su fuerte tirón de consumo energético en la base de la recuperación del petróleo y sus derivados, que logran aumentar en 2.001 ktep $(4,2 \%)$. También ha intervenido el sector del transporte y el incremento del parque de turismos con motor Diesel. Sin embargo, en el sector doméstico y terciario disminuyó la demanda de estos productos para calefacción, ante la progresiva sustitución por gas natural.

El carbón disminuyó en un $-5,4 \%$ respecto a 1996 . Su consumo se concentra sobre todo en dos sectores industriales: siderurgia y fábricas de cemento. Ambos representan más del $80 \%$ del carbón consumido. El $20 \%$ restante corresponde a otras industrias y al sector residencial. Tanto en siderurgia como en cementeras disminuyó la demanda de carbón $(-3,7 \%$ y $-29,4 \%$, respectivamente), pese a un aumento de su actividad. Lo que ha sucedido en realidad es que el carbón continúa siendo sustituido por otros combustibles, especialmente el coque de petróleo.

Las energías renovables pasaron de $3.271 \mathrm{ktep}$ a $3.299 \mathrm{ktep}(0,9 \%)$, lo que supone una variación poco significativa. En conjunto, aportaron el $4,3 \%$ a la estructura del consumo final en España en 1997, tal y como se observa en el Cuadro 2. 
Cuadro 2. Consumo de energía final. 1996-1997

\begin{tabular}{lrrrrr}
\hline Fuente & \multicolumn{2}{c}{1996} & \multicolumn{2}{c}{1997} & Variación \\
& \multicolumn{1}{c}{ Ktep } & \multicolumn{1}{c}{$\%$} & \multicolumn{1}{c}{ ktep } & \multicolumn{1}{c}{$\%$} & \multicolumn{1}{c}{$\%$} \\
\hline Carbón & 2.464 & 3,3 & 2.330 & 3,0 & $-5,4$ \\
Productos petrolíferos & 48.107 & 65,0 & 50.108 & 64,8 & 4,2 \\
Gas natural & 7.325 & 9,9 & 8.212 & 10,6 & 12,1 \\
Electricidad & 12.843 & 17,4 & 13.364 & 17,3 & 4,1 \\
Energias renovables & 3.271 & 4,4 & 3.299 & 4,3 & 0,9 \\
Total & 74.010 & 100 & 77.313 & 100 & 4,5 \\
\hline
\end{tabular}

Ktep: miles de toneladas equivalentes de petróleo.

Fuente: Ministerio de Industria y Energía.

El Cuadro 3 resume el consumo de energía final por sectores. El aumento se registra en los sectores productivos (industria y transporte), ya que en los denominados "usos diversos" el descenso es considerable. Muy especialmente, el incremento del consumo ha sido muy alto en el transporte, que representa en 1997 el 39,5\% del consumo de energía final, y consigue desplazar a la industria al segundo puesto.

Cuadro 3. Consumo de energía final por sectores. 1996-1997

\begin{tabular}{lccccc}
\hline Sector & \multicolumn{2}{c}{1996} & \multicolumn{2}{c}{1997} & Variación \\
& Ktep & $\%$ & ktep & $\%$ & \multicolumn{1}{c}{$\%$} \\
\hline Industria & 28.034 & 37,8 & 30.225 & 39,0 & 7,8 \\
Transportes & 27.461 & 37,0 & 30.644 & 39,5 & 11,5 \\
Usos diversos & 18.735 & 25,2 & 16.622 & 21,5 & $-11,3$ \\
Total & 74.230 & 100 & 77.491 & 100 & 4,4 \\
\hline
\end{tabular}

Ktep: miles de toneladas equivalentes de petróleo.

Fuente: Ministerio de Industria y Energía.

\subsection{Producción de energía primaria}

La producción nacional de energía primaria fue en el año 1997 de $31.086 \mathrm{ktep}$, lo que entraña un descenso de $1.208 \mathrm{ktep}$ respecto a 1996. Salvo el ejemplo de las energias renovables, todas las demás fuertes energéticas experimentan descensos de mayor a menor intensidad. Destacan el gas natural y el petróleo, con un $-62,4 \%$ y un $-41,2 \%$, respectivamente, lo que indica el progresivo agotamiento de los yacimientos 
españoles. La producción de energía hidráulica (la única renovable que presenta un balance negativo) bajó un $-12,2 \%$ entre ambas fechas, al igual que la nuclear $(-1,8 \%)$ y el carbón $(-1,9 \%)$. La producción de carbón representa casi la tercera parte de toda la producción energética nacional, sólo superada por la nuclear.

Cuadro 4. Producción nacional de energía primaria. 1996-1997

\begin{tabular}{lrrrrr}
\hline Fuente & \multicolumn{2}{c}{1996} & \multicolumn{2}{c}{1997} & \multicolumn{1}{c}{ Variación } \\
& \multicolumn{1}{c}{ Ktep } & \multicolumn{1}{c}{$\%$} & \multicolumn{1}{c}{$k$ ktep } & $\%$ & \multicolumn{1}{c}{$\%$} \\
\hline Carbón & 9.653 & 29,9 & 9.473 & 30,5 & $-1,9$ \\
Petróleo & 519 & 1,6 & 305 & 1,0 & $-41,2$ \\
Gas natural & 413 & 1,3 & 155 & 0,5 & $-62,4$ \\
Hidráulica & 3.488 & 10,8 & 3.064 & 9,9 & $-12,2$ \\
Nuclear & 14.680 & 45,5 & 14.411 & 46,4 & $-1,8$ \\
Energias renovables & 3.541 & 11,0 & 3.678 & 11,8 & 3,9 \\
Total & 32.294 & 100 & 31.086 & 100 & $-3,7$ \\
\hline
\end{tabular}

Ktep: miles de toneladas equivalentes de petróleo.

Fuente: Ministerio de Industria y Energía.

Debido al descenso en la producción interior, el grado de dependencia energética aumentó en España entre 1996 y 1997. Junto al mal año hidráulico, la situación se explica por un fuerte tirón en la demanda de energias procedentes del exterior, especialmente petróleo y gas natural. La dependencia española, expresada en porcentajes, pasó del 68,1\% en 1996 al $71 \%$ en 1997, lo que representa unas tasas muy elevadas que obligan a importar grandes cantidades de energía para poder cubrir la demanda interna. En comparación con la dependencia que presenta el conjunto de países de la Unión Europea, que se sitúa en torno al $45-50 \%$, la dependencia española del exterior es excesiva y supone un importante riesgo ante cualquier problema que pueda surgir en los mercados energéticos internacionales.

La dependencia energética, que se calcula relacionando producción interior y consumo total de energía, está próxima al $100 \%$ en el caso del petróleo y gas natural, mientras que en el carbón la cifra es del $46,2 \%$. En el caso de las energías nuclear, hidráulica y renovables, el autoabastecimiento es total. Las cifras aparecen recogidas en el Cuadro 5. 
Cuadro 5. Dependencia energética española (\%). 1996-1997

\begin{tabular}{lrr}
\hline Fuente & 1996 & 1997 \\
\hline Carbón & 37,9 & 46,2 \\
Petróleo & 99,1 & 99,5 \\
Gas natural & 95,1 & 98,6 \\
Nuclear & 0 & 0 \\
Hidráulica & 0 & 0 \\
Renovables & 0 & 0 \\
Total & 68,1 & 71,0 \\
\hline
\end{tabular}

Fuente: Ministerio de Industria y Energía y elaboración propia.

\section{SECTORES ENERGÉTICOS}

En este apartado se analizan las principales variables que han definido la evolución de la energía en España a través de sus diferentes sectores. Cada uno de ellos ha mantenido su propia dinámica y es lo que pasamos a comentar a continuación, tanto para el carbón como para el sector eléctrico, el nuclear, gas natural y petróleo. Las energías renovables constituyen el objetivo del siguiente apartado, ya que merecen un estudio individualizado debido al extraordinario empuje que están experimentando en nuestro país.

Aunque en general, la producción de carbón tuvo un crecimiento negativo de $-1,9 \%$ entre 1996 y 1997, la hulla y la antracita crecieron en la última fecha en comparación con la anterior. La producción de lignito negro se mantuvo en cifras muy parecidas, mientras que la de lignito pardo disminuyó en un $-11,7 \%$. Este descenso se explica por la mayor utilización de carbón importado ante la mejora planificada de las emisiones en varias centrales térmicas, como las de Meirama y As Pontes.

El número total de empresas dedicadas a la extracción de carbón fue en 1997 de 80. La mano de obra empleada ascendía a 47.383 trabajadores, con un descenso global de 4.815 empleados y uno relativo del $9,2 \%$.

El sector del carbón presenta una estructura caracterizada por las empresas grandes. El 28\% de las mismas tuvieron en 1997 una producción superior a las 200.000 toneladas al año y concentraron alrededor del $82 \%$ de la producción total de hulla, antracita y lignito negro, y el $85 \%$ de los empleados. El $72 \%$ restante son empresas pequeñas que producen me- 
nos de las 200.000 toneladas anuales: generan el $18 \%$ de la producción y concentran el $15 \%$ de los trabajadores empleados en el sector.

Los cálculos efectuados sobre los recursos nacionales de carbón arrojan una cifra de 4.317 millones de toneladas, de las que 2.299 corresponden a hulla y antracita y el resto a los lignitos, tanto negro como pardo. La cifra total se desagrega en cuatro grupos dependiendo de la menor o mayor probabilidad de error en su estimación. Así aparecen los tonelajes "muy probables", "probables", "posibles" e "hipotéticos" o "especulativos". Los tres primeros se consideran recursos identificados y reservas explotables desde el punto de vista geológico. En cuanto a los recursos hipotéticos, hay indicios de su existencia, pero no están bien identificados.

Cuadro 6. Recursos españoles de carbón, por tipos (millones de toneladas)

\begin{tabular}{lcrrrr}
\hline Tipo de carbón & Muy probables & Probables & Posibles & Hipotéticos & \multicolumn{1}{c}{ Total } \\
\hline Hulla y antracita & 428,1 & 359,7 & 396,1 & $1.116,0$ & $2.299,9$ \\
Lignito negro & 318,6 & 206,4 & 209,0 & 747,0 & $1.481,0$ \\
Lignito pardo & 403,1 & 133,5 & - & - & 536,6 \\
Total & $1.149,8$ & 699,6 & 605,1 & $1.863,0$ & $4.317,5$ \\
\hline
\end{tabular}

Fuente: Garcia Alonso e Iranzo Martín, 1989.

Los recursos totales de carbón de nuestro país garantizan los actuales niveles de producción durante más de un siglo, y esto sin tener en cuenta la posibilidad de nuevos descubrimientos de yacimientos. Estos recursos están muy concentrados espacialmente: más de las $2 / 3$ partes del carbón español se sitúan a ambos lados de la Cordillera Cantábrica (León con el $35,4 \%$ y Asturias con el $29,2 \%$ ). A continuación sigue Teruel con el $17,2 \%$. Por tipos de carbón, la concentración también es muy alta: León y Asturias tienen el $91 \%$ del total de hulla y antracita, Teruel el $70 \%$ del lignito negro y La Coruña el $84 \%$ del lignito pardo.

Si bien las cifras de recursos carboníferos son importantes, los carbones españoles son escasamente competitivos debido a los altos costes de explotación de los yacimientos. Si se mantienen los elevados precios del petróleo de la actualidad, puede resultar más competitiva la extracción de los carbones nacionales, pero esto se puede modificar en un futuro próximo y alterar cualquier tipo de expectativa al respecto. 
El consumo final de electricidad creció en 1997. La estructura de generación muestra un importante descenso de la energía hidroeléctrica y un aumento en carbón y gas natural. El fuerte aumento del gas natural se debe a la entrada en funcionamiento de la central por gasificación de Elcogás y a la transformación de centrales de fuelóleo a gas. La producción en centrales nucleares bajó ligeramente, aunque todavía en 1997 aportaban casi el $30 \%$ de la electricidad nacional.

Cuadro 7. Balance eléctrico nacional según origen. 1996-1997 (Gigawatios hora)

\begin{tabular}{lrrrrr}
\hline Fuente & \multicolumn{2}{c}{1996} & \multicolumn{2}{c}{1997} & Variación \\
& GWh & $\%$ & \multicolumn{1}{c}{ GWh } & $\%$ & \multicolumn{1}{c}{$\%$} \\
\hline Hidroeléctrica & 41.341 & 23,4 & 36.742 & 19,4 & $-11,1$ \\
Nuclear & 56.329 & 31,8 & 55.298 & 29,2 & $-1,8$ \\
Carbón & 54.957 & 31,1 & 64.207 & 33,9 & 16,8 \\
P. petrolíferos & 14.032 & 7,9 & 13.645 & 7,2 & $-2,8$ \\
Gas natural & 8.103 & 4,6 & 16.299 & 8,6 & 101,2 \\
Otros & 1.634 & 0,9 & 2.239 & 1,2 & 37,0 \\
$\quad$ Producción bruta & 176.396 & 100 & 188.430 & 100 & 6,8 \\
$\quad$ Consumos & 6.965 & 7.995 & & & 14,8 \\
$\quad$ Producción neta & 169.431180 .435 & & & 6,5 \\
\hline
\end{tabular}

'Eólica, residuos sólidos urbanos y otros combustibles.

Fuente: Ministerio de Industria y Energía.

El potencial hidroeléctrico español se ve afectado por el problema de la irregularidad del régimen de lluvias. La zona norte recibe precipitaciones a lo largo de todo el año, mientras que hacia el sur se prolongan los periodos de sequía. La irregularidad no sólo es estacional sino también interanual, obligando a la construcción de costosas obras para regular los caudales y poder acumular agua en los años lluviosos y cederla en los secos. Estas irregularidades neutralizan las favorables condiciones topográficas para la producción hidroeléctrica en nuestro país.

España cuenta con uno de los parques hidroeléctricos más importantes del mundo, sólo superado por países de gran tamaño (EE.UU., Canadá o Rusia) o muy montañosos y con fuertes precipitaciones (Noruega y Suecia). La potencia hidráulica instalada sigue todavía muy concentrada, ya que en torno al $65 \%$ de la misma se localiza en las cuencas Norte, Duero y Tajo, destacando las provincias de Cáceres, Salamanca, Orense 
y Lérida. Algo más de la cuarta parte corresponde a centrales de bombeo, que son un tipo de central hidráulica muy eficaz en cuanto a la utilización del agua como recurso energético.

Estas centrales disponen de dos embalses situados a diferente altura. Cuando la demanda eléctrica es mayor, la central superior funciona como cualquier planta hidroeléctrica, turbinando agua para la producción de electricidad, agua que luego es retenida en el embalse inferior. En los periodos de menor consumo, se bombea el agua desde el embalse inferior al superior, para poder atender en mejores circunstancias el incremento posteror de la demanda. La gran ventaja de las centrales de bombeo es que permiten multiplicar el uso de los caudales hídricos, ya que las operaciones de bombeo y turbinado se pueden repetir indefinidamente. Esto eleva el potencial hidroeléctrico aprovechable y garantiza una explotación más eficaz y racional. Las centrales de bombeo pueden ser mixtas, al combinar el agua que se transfiere del embalse inferior al superior con el agua que procede directamente del río, o de bombeo puro, en las que existe un embalse superior artificial localizado fuera del cauce fluvial. Ejemplos de centrales mixtas con bombeo en España son las de Aldeadávila (Salamanca), Almendra (Salamanca), Bao (Orense), As Portas (Orense) y Valdecañas (Cáceres). De bombeo puro son las de Sallent (Lérida), Tajo de la Encantada (Málaga), Mediajo (Cantabria) y Bolarque (Guadalajara).

En 1998, España contaba con un total de 9 centrales nucleares en funcionamiento, situadas en 7 emplazamientos y con una potencia instalada de $7.638 \mathrm{MW}$, lo que representa el $15 \%$ de la potencia total instalada en España. El factor de carga (relación entre la energía eléctrica producida en un periodo de tiempo y la que se hubiera podido producir en ese mismo periodo funcionando a la potencia nominal) del parque nuclear español durante 1998 ha sido del $88,4 \%$, cifra que se sitúa entre las más altas del mundo y que se ha alcanzado a pesar de la parada de unos tres meses que tuvo que efectuar la central nuclear de Trillo para sustituir una parte del alternador que resultó averiado.

En la primera parte del ciclo del combustible nuclear destaca la producción de la planta Quercus de concentrados de uranio y que la Empresa Nacional del Uranio, S.A. (ENUSA) tiene en la provincia de Salamanca, ascendiendo en 1997 a 300 toneladas de $\mathrm{U}_{3} \mathrm{O}_{8}$. En cuanto a los suministros, en ese año se acordó con la empresa surafricana Nucfor el suministro de concentrados de uranio para el periodo 2001 al 2005 por un total de 1.306 toneladas de $\mathrm{U}_{3} \mathrm{O}_{8}$ durante los cinco años, así como con 
la empresa Internexco para el suministro de concentrados de uranio rusos (472 toneladas), para entregar después del 2002. Al mismo tiempo, toda una serie de contratos con otras empresas y paises aseguran las necesidades en un $90 \%$ hasta el 2006.

La potencia nominal del parque nuclear español en 1998 se incrementó en $57,6 \mathrm{MW}$, pasando a ser los $7.638 \mathrm{MW}$ ya comentados. Por centrales, los incrementos han sido los siguientes:

- CN Cofrentes: 35,4 MW, con una potencia total de 1.025,4 MW.

- CN Santa María de Garoña: $6 \mathrm{MW}$; potencia total: $466 \mathrm{MW}$.

- CN Ascó I: $6 \mathrm{MW}$; potencia total: $979 \mathrm{MW}$.

- CN Ascó II: 10,2 MW; potencia total: 976,2 MW.

Cuadro 8. Potencia eléctrica y producción de origen nuclear. 1998

\begin{tabular}{lccc}
\hline Central & Tipo & Entrada en servicio & Potencia instalada(MW) \\
\hline José Cabrera & PWR & 1968 & 160 \\
Garoña & BWR & 1971 & 466 \\
Almaraz I & PWR & 1981 & 974 \\
Ascó I & PWR & 1983 & 979 \\
Almaraz II & PWR & 1983 & 983 \\
Cofrentes & BWR & 1984 & 1.025 \\
Ascó II & PWR & 1985 & 976 \\
Vandellós II & PWR & 1987 & 1.009 \\
Trillo I & PWR & 1988 & 1.066 \\
Total & & & 7.638 \\
\hline
\end{tabular}

PWR: reactor de agua a presión; BWR: reactor de agua en ebullición.

Fuente: Ministerio de Industria y Energía.

A comienzos de 1998 se autorizó a la empresa ENRESA a la ejecución de las actividades de desmantelamiento de la central Vandellós I. Esta fue la primera autorización de desmantelamiento de una central concedida en nuestro país, operación que ha consistido en la demolición de edificios y estructuras, excepto el cajón del reactor, que quedará aislado durante unos 5 años y vigilado desde un punto de vista ambiental y radiológico. Transcurridos unos 30 años, comenzará a desmantelarse el cajón así como el propio reactor, quedando liberado totalmente el emplazamiento de la central y pudiendo utilizarlo sin ninguna clase de limitaciones. 
La incorporación del gas natural en España se llevó a cabo con un cierto retraso respecto a otros países europeos, que lo hacen en los años 50 y 60. En ello influyó el alejamiento de los principales yacimientos del continente, la imposibilidad del transporte marítimo del gas hasta 1964 y las favorables perspectivas del mercado petrolífero internacional hasta principios de los 70. En 1972 se crea la Empresa Nacional del Gas (ENAGAS), con una participación del INI del 100\% de su capital. La producción nacional comenzó en 1984 en Serrablo (Huesca) y en 1986 comenzó la explotación del campo "Gaviota", frente a la costa vizcaína; se trata, por lo tanto, éste último de un yacimiento "off shore" que está unido con dos tuberías submarinas a la planta de tratamiento situada en Bermeo. Sus reservas representan aproximadamente el $75 \%$ de todas las conocidas a nivel nacional.

Al margen de la producción nacional, el sector del gas natural continúa creciendo, pasando de representar el $7,6 \%$ de toda la energía primaria en 1995 al $10,3 \%$ en 1997 . Esta evolución acabará por aproximar a nuestro país a la media europea, que se sitúa en torno al $20 \%$. El mayor consumo de gas natural lo representa el mercado industrial, que experimentó una fuerte subida de 1996 a 1997. En conjunto, este mercado supone el $68 \%$ de todo el consumo de gas, seguido del perteneciente al ámbito doméstico-comercial (15\% de la demanda). En otros países europeos, con condiciones climáticas más desfavorables y con una red de distribución más extensa, el mercado doméstico-comercial se aproxima al $40 \%$ de la demanda total de gas natural. El uso de esta fuente de energía para generación eléctrica subió del $2 \%$ en 1996 al $12 \%$ de las ventas totales en 1997.

Las importaciones de gas han evolucionado a la par que la demanda. El principal país suministrador es Argelia, seguido de Noruega, Libia, Abu Dhabi y Qatar. El aprovisionamiento nacional sólo significa en torno al $1 \%$ del total. La infraestructura gasista cuenta en España con unos 9500 kilómetros de longitud y están previstas futuras ampliaciones. En 1996 se inauguró el gasoducto Magreb-Europa, transportando desde entonces casi el $40 \%$ de nuestros aprovisionamientos del exterior.

De Oriente Medio y del norte de Africa procede la mayoría del petróleo importado. La producción nacional es insignificante, aunque se ha procedido a realizar en los últimos años una importante actividad exploratoria en España. De los nueve sondeos llevados a cabo en 1997, cuatro dieron resultado positivo.

En cuanto a la demanda de los diferentes productos petrolíferos, aumentan las gasolinas sin plomo, los gasóleos de automoción, el querose- 
no para la aviación y el coque de petróleo, éste último debido al incremento de la actividad en diversos sectores industriales (aceros especiales, cementeras e industrias del aluminio). Por otro lado, disminuye el consumo de las gasolinas con plomo, como consecuencia de la modernización del parque automovilístico, y el de los gasóleos para calefacción ante la progresiva introducción de gas natural en la calefacción doméstica. En conjunto, el consumo de productos petrolíferos durante 1997 fue de 56,4 millones de toneladas, con un aumento del $5 \%$ frente a los 52,7 millones de toneladas en 1996.

Durante 1997 se reactivó la investigación en el campo de la producción de hidrocarburos en España y como resultado fue el descubrimiento de crudo en el son de Boquerón y la puesta en producción del Campo Poseidón. El sondeo Boquerón está situado a 4.654 metros de profundidad, en formaciones de carbonatos mesozoicos. A finales de año, la producción se situaba en torno a los 5.500 barriles/día. El Campo Poseidón está situado en el subsuelo marino del Golfo de Cádiz. Está formado por dos yacimientos de gas natural a unos 1.500 metros bajo el mar y con unas reservas estimadas en unos 1.700 millones de metros cúbicos para el conjunto de los dos yacimientos. Para su puesta en explotación se han construido unos 65 kilómetros de gasoductos hasta tierra, en donde continúa hasta una planta de tratamiento de gas para la separación de líquidos, filtrado, compresión, secado, medida y odorización. El gas así tratado es posteriormente conducido a la red básica para su distribución.

\section{LAS ENERGIAS RENOVABLES}

En 1998, el conjunto de las energías renovables aportó el 6,3\% del balance energético nacional, con un consumo total en este campo de 7.173 ktep. Las principales fuentes renovables son la biomasa (3.644 ktep) y la hidráulica (3.127 ktep). El resto (residuos sólidos urbanos, solar fotovoltaica y térmica, geotermia y eólica) presentan producciones aún muy reducidas, aunque en el terreno de la energía eólica se están produciendo fuertes aumentos. De hecho, consiguió duplicar su aportación respecto al año anterior.

La producción eléctrica con energías renovables contaba con una potencia instalada en 1998 de $18.856 \mathrm{MW}$, que produjeron $39.537 \mathrm{GWh}$. La energía hidráulica es la más importante, ya que aporta el $94 \%$ de la potencia instalada y el $92 \%$ de la producción. 
Cuadro 9. Producción eléctrica con energías renovables. 1998

\begin{tabular}{lrr}
\hline Fuente & Potencia (MW) & Producción (GWh/año) \\
\hline Hidráulica & $17.730,6$ & $36.360,4$ \\
Biomasa & 188,8 & $1.139,1$ \\
Residuos sólidos urbanos & 94,1 & 585,8 \\
Eólica & 834,1 & $1.437,0$ \\
Solar fotovoltaica & 8,7 & 15,3 \\
Total & $18.856,3$ & $39.537,6$ \\
\hline
\end{tabular}

Fuente: Instituto para la Diversificación y Ahorro de la Energía.

La producción térmica con energías renovables fue de 3.505,9 ktep, de los que el $99 \%$ fueron aportados por el uso de la biomasa. La energía solar térmica se aprovecha sobre todo en instalaciones de baja temperatura para producción de agua caliente sanitaria en el sector doméstico y de servicios, como hoteles y colegios. La energía geotérmica aporta una pequeña cantidad en pequeñas instalaciones de baja temperatura, en general para usos turísticos y agrícolas.

La energía minihidráulica, que se refiere a las minicentrales de potencia igual o menor a $10 \mathrm{MW}$, es un sector tradicional de producción eléctrica en nuestro país, con 1.024 centrales en funcionamiento y una potencia de $1.510 \mathrm{MW}$. La actividad del área minihidráulica está bastante repartida por todas las Comunidades Autónomas, destacando Castilla y León, Cataluña y Andalucia. Por cuencas hidrográficas destacan las del Ebro, Norte y Duero.

La biomasa está integrada por los cultivos energéticos, la biomasa residual, el biogás y los biocarburantes y constituye por su aprovechamiento la principal fuente de recursos renovables. Esta fuente, además, se relaciona con un gran número de sectores: agricultura (cultivos energéticos), transportes (biocarburantes), sector doméstico e industrial (residuos), etc. El doméstico consumió el $54,6 \%$ de toda la biomasa en España.

Andalucía, Galicia y Castilla y León representan conjuntamente el $50 \%$ de la biomasa. Siguen en importancia Cataluña y Asturias, cuyos consumos superan las 200.000 tep al año. 
Cuadro 10. Consumo de biomasa por Comunidades Autónomas. 1998

\begin{tabular}{lrc}
\hline CC.AA. & Total (tep) & Porcentaje \\
\hline Galicia & 650.947 & 17,8 \\
Asturias & 243.300 & 6,7 \\
Cantabria & 48.909 & 1,3 \\
País Vasco & 180.190 & 4,9 \\
Navarra & 114.029 & 3,2 \\
La Rioja & 34.890 & 0,9 \\
Aragón & 177.423 & 4,9 \\
Cataluña & 289.502 & 7,9 \\
Castilla y León & 420.286 & 11,5 \\
Madrid & 104.798 & 2,9 \\
Extremadura & 110.452 & 3,0 \\
Castilla-La Mancha & 179.731 & 4,9 \\
Comunidad Valenciana & 189.765 & 5,2 \\
Andalucia & 783.378 & 21,5 \\
Murcia & 64.773 & 1,8 \\
Baleares & 49.801 & 1,5 \\
Canarias & 2.608 & 0,1 \\
Total & 3.644 .782 & 100 \\
\hline
\end{tabular}

Fuente: Instituto para la Diversificación y Ahorro de la Energía y elaboración propia.

La producción de residuos sólidos urbanos durante 1996 supuso unos 15 millones de toneladas, es decir, 1,06 kilogramos por habitante y día, cifra que aumenta progresivamente. Estos residuos pueden teóricamente proporcionar una energía próxima a los 2,5 Mtep al año. A finales de 1998 habia un total de nueve plantas de valorización energética de residuos, que incineraron 1,2 millones de toneladas de basuras y contaban con una potencia eléctrica instalada de $94,1 \mathrm{MW}$; la producción bruta de energía eléctrica fue de $586 \mathrm{GWh} / \mathrm{año}$.

La energía eólica está experimentando un fuerte aumento en los últimos años, con 834 MW de potencia instalada en 1998, lo que sitúa a España en tercer lugar dentro de la Unión Europea después de Alemania y Dinamarca. La tecnología nacional para la construcción de máquinas eólicas está plenamente desarrollada, sobre todo en el tramo de máquinas de media potencia. Este sector está formado aproximadamente por 200 empresas, entre fabricantes, suministradores, obra civil, etc. En 1998 se invirtieron más de 56.000 millones de pesetas, pudiéndose haber llegado 
en 1999 a los 135.000 millones (según previsiones de finales de 1998). A nivel nacional, la eólica se concentra sobre todo en Navarra, Galicia y Aragón, que representan en conjunto más del $71 \%$. Siguen en importancia Andalucía, Canarias y Castilla y León.

España cuenta con significativos recursos para un amplio aprovechamiento de la energía solar, cuya radiación oscila entre 3,2 y 5,3 $\mathrm{KWh} /$ metro cuadrado/día. El mercado potencial de utilización de la solar térmica es amplio, y la mayor parte corresponde a viviendas y sector hotelero. A finales de 1998, la superficie total instalada en España era de 340.844 metros cuadrados, destacando Andalucía, Baleares, Canarias y Madrid, que representan en conjunto las tres cuartas partes del total nacional. En ese año se finalizaron numerosos proyectos de aplicaciones de baja temperatura con una inversión que superó los 1.500 millones de pesetas. Las cifras son aún modestas para las posibilidades del mercado y la situación en países de nuestro entorno.

La energía solar fotovoltaica, a pesar del fuerte incremento experimentado en los últimos diez años, ocupa un lugar poco destacado en el panorama general de las energías renovables. Las inversiones en 1998 superaron los 3.000 millones de pesetas y el tejido industrial está compuesto por 73 empresas, entre fabricantes, empresas instaladoras y de comercialización. Debido a que la capacidad de producción de módulos fotovoltaicos supera las necesidades del mercado interior, la exportación es importante en este sector energético.

Los recursos geotérmicos existentes en España son escasos y, en general, de baja temperatura. Su aprovechamiento está muy localizado en centros balnearios, en donde se asocia el uso de las aguas termales al energético para calefacción y climatización de piscinas. A corto plazo no cambiarán las perspectivas de esta fuente debido a la escasez de recursos y a las dificultades para su utilización.

Desde que entró en vigor el Programa de Energías Renovables para el periodo 1991-2000, el número de proyectos finalizados supera los 8.000, con una inversión total de 270.476 millones de pesetas. En 1998, los proyectos de energías renovables puestos en funcionamiento son los que figuran en el cuadro adjunto, teniendo en cuenta que el número de instalaciones es mayor que el de proyectos, ya que mediante un proyecto pueden realizarse un grupo de instalaciones. 
Cuadro 11. Proyectos de energías renovables puestos en funcionamiento en 1998

\begin{tabular}{lccc}
\hline Fuente & № proyectos & Energia (tep) & Inversión total (mill.pts.) \\
\hline Minihidráulica & 22 & 12.682 & $6.261,9$ \\
Biomasa & 19 & 22.607 & $1.540,0$ \\
R.S.U. & 1 & 1.800 & 224,6 \\
Eólica & 229 & 86.530 & $56.515,7$ \\
Solar fotovoltaica & 766 & 192 & $3.189,8$ \\
Solar térmica & 1.887 & 1.514 & $1.505,8$ \\
Total & 2.924 & 125.325 & $69.237,8$ \\
\hline
\end{tabular}

Fuente: Instituto para la Diversificación y Ahorro de la Energía.

\section{POLÍTICA ENERGÉTICA}

En el marco de la estrategia comunitaria de política energética, en España ha habido una clara orientación hacia la liberalización y la regulación de la competencia. Esta política ha ido acompañada por actuaciones puntuales de privatización de empresas públicas, como Endesa, enajenada por parte de la SEPI mediante la oferta pública de venta de acciones.

En 1997 se aprobó la Ley 54/97 del Sector Eléctrico, en la que se regula el sector, se reduce al mínimo la intervención estatal y se garantiza el suministro con la calidad adecuada. La producción de energía eléctrica pasa a funcionar bajo el principio de libre competencia.

En cuanto a la energía nuclear destaca una amplia normativa sobre funcionamiento de centrales, instalaciones radiactivas y autorizaciones de puesta en marcha o modificación de equipos, todo ello bajo la base jurídica del Tratado de No Proliferación Nuclear (TNP).

En 1998 se aprobó la Ley de Hidrocarburos (Ley 34/98), encargada de iiberalizar el sector del gas natural y de atender la creciente demanda de gas en España en un marco de mercado en régimen de competencia. Lo mismo cabe decir del sector del petróleo. La normativa más reciente ha ido dirigida en este caso a la distribución al por menor de carburantes y combustibles petroliferos en instalaciones de venta al público.

Una parte importante de la política energética lo constituye el apoyo prestado al fomento y desarrollo de las energías renovabies, que opti- 
mizan los recursos naturales y mejoran las condiciones medioambientales. Todo esto toma como punto de referencia el Plan de Ahorro y Eficiencia Energética (PAEE), cuyos objetivos se han extendido desde 1991 al 2000. En cuanto a inversión, a finales de 1997 estaban cumplidos el $107 \%$ de los objetivos finales. El Plan también ha supuesto una importante reducción de emisiones atmosféricas, próximas a las 19.200 toneladas de $\mathrm{CO}_{2}, 57$ toneladas de $\mathrm{NO}_{x}$ y 397 toneladas de $\mathrm{SO}_{2}$ a finales de 1997. Dentro del Plan, el Programa de Energías Renovables se ha cumplido en un $207 \%$ en cuanto a potencia instalada y en un $234 \%$ en producción de energía eléctrica, destacando sobre todo la eólica.

La elaboración del Plan de Fomento de las Energias Renovables para el periodo 1999-2010 será el que provoque un nuevo ritmo en el crecimiento de estas fuentes energéticas, al amparo del Libro Blanco de Energías Renovables adoptado por la Comisión Europea en 1997, y cuya estrategia se dirige a duplicar hasta el 2010 la participación de estas fuentes, pasando del $6 \%$ inicial al $12 \%$ al final del periodo.

El apoyo a la investigación en este campo ha permitido realizar proyectos de desarrollo tecnológico en el área de energía y medio ambiente para la mejora de la calidad ambiental de los combustibles fósiles, incrementar la competitividad de las energías renovables, avanzar en todo lo referente a seguridad nuclear, etc., muchos de los cuales se han llevado a cabo en el denominado Centro de Investigaciones Energéticas, Medioambientales y Tecnológicas (CIEMAT).

Una de las líneas principales de investigación de este organismo es el del impacto ambiental de la energía, con estudios como el de "Protección radiológica por intervención", en el que se realiza una correcta evaluación del riesgo de la población en situación de contaminación radiactiva. Al mismo tiempo, se ha avanzado en el desarrollo de estrategias para recuperar áreas contaminadas tras un accidente nuclear. En el estudio titulado "Impacto radiológico ambiental" se analizan los efectos de los almacenamientos de residuos radiactivos.

En relación con las energías renovables, las líneas de trabajo del CIEMAT son muy diversas y heterogéneas. Debido al carácter discontinuo y aleatorio de estas fuentes es muy importante conocer las posibilidades de aprovechamiento, sobre todo en aquellos casos de fuerte desarrollo tecnológico e implantación en el mercado, como ocurre con 
la energía solar y eólica. En este sentido destaca el proyecto de "Evaluación de recursos de energías renovables". En el campo de la eólica, el trabajo ofrece nuevas herramientas para evaluar y caracterizar el potencial eólico y para la selección de emplazamientos de aerogeneradores. En cuanto a la energía solar, se ha avanzado en la determinación de los recursos de radiación global y radiación directa. Destaca igualmente la elaboración del Mapa de Radiación Solar de Andalucía a partir del tratamiento de imágenes del satélite Meteosat, así como la participación en varios estudios de ámbito europeo en la Planta Solar de Almería, en la Central Solar Fotovoltaica Sierra de María y en la Central Fotovoltaica Toledo PV.

\section{ENERGIA Y MEDIO AMBIENTE}

La opinión de la sociedad española sobre los problemas del medio ambiente gira en torno a la desertización, la falta de agua y su gestión o los incendios forestales, pero poco en cuanto a las relaciones entre medio ambiente y energía. Hay que advertir, en cualquier caso, que éstas últimas plantean en nuestro país situaciones menos graves que las sufridas, por ejemplo, en países del centro de Europa.

La demanda social de reducción de las emisiones de $\mathrm{CO}_{2}$ es innegable en cualquier caso y ello ha impulsado el uso de energías renovables, que vuelven a plantearse como alternativa, ahora con un claro objetivo medioambiental; tras las crisis petrolíferas de los años setenta, empezaron a ser consideradas como posible solución a una supuesta falta de aprovisionamiento de energía.

El cambio climático parece estar estrechamente relacionado con el consumo energético, aunque hay quien discute dicha vinculación. En 1998 se creó en España el Consejo Nacional del Clima (Real Decreto $177 / 1998$ de 16 de Febrero) y dentro del mismo, junto a otros grupos de trabajo, se constituyó el grupo de Industria y Energía. Según las previsiones del grupo, el porcentaje de incremento de las emisiones de gases de efecto invernadero será de un $21 \%$ entre el año 1990 y el 2010. Sin embargo, para alcanzar los objetivos del Protocolo de Kioto, ese aumento debe descender al 15\%, según acuerdo del Consejo de Ministros de Medio Ambiente de la Unión Europea de junio de 1998. 
Cuadro 12. Emisiones energéticas de $\mathrm{CO}_{2}$ en la Unión Europea (Mt)

\begin{tabular}{lrrrrrrr}
\hline Pais & 1990 & 1993 & 1994 & 1995 & 1996 & $\begin{array}{c}1990-1996 \\
\%\end{array}$ & $\begin{array}{c}1990-2010 \\
\%\end{array}$ \\
\hline Alemania & 957,1 & 877,8 & 854,8 & 866,2 & 888,8 & $-7,1$ & $-21,0$ \\
Austria & 55,0 & 54,3 & 54,1 & 56,7 & 57,8 & 5,1 & $-13,0$ \\
Bélgica & 104,5 & 106,3 & 110,5 & 111,0 & 116,7 & 11,7 & $-7,5$ \\
Dinamarca & 52,7 & 58,5 & 62,6 & 59,9 & 73,9 & 40,3 & $-21,0$ \\
España & 202,0 & 210,4 & 221,2 & 226,7 & 224,7 & 11,2 & 15,0 \\
Finlandia & 51,6 & 55,2 & 58,9 & 56,2 & 60,0 & 16,3 & 0,0 \\
Francia & 352,4 & 348,8 & 334,0 & 345,4 & 364,0 & 3,3 & 0,0 \\
Grecia & 70,9 & 73,1 & 75,4 & 77,0 & 81,7 & 15,2 & 25,0 \\
Holanda & 153,0 & 164,2 & 160,5 & 166,8 & 177,7 & 16,1 & $-6,0$ \\
Irlanda & 30,2 & 30,8 & 32,2 & 32,3 & 34,9 & 15,6 & 13,0 \\
Italia & 388,6 & 384,1 & 380,4 & 403,2 & 399,1 & 2,7 & $-6,5$ \\
Luxemburgo & 10,6 & 11,3 & 10,7 & 8,7 & 8,9 & $-16,2$ & $-28,0$ \\
Portugal & 39,1 & 43,7 & 44,4 & 48,0 & 45,6 & 16,7 & 27,0 \\
Reino Unido & 566,9 & 546,7 & 537,0 & 531,3 & 550,2 & $-2,9$ & $-12,5$ \\
Suecia & 50,6 & 51,1 & 54,1 & 53,6 & 58,4 & 15,4 & 4,0 \\
U.E. & 3085,2 & 3016,3 & 2990,8 & 3043,9 & 3142,4 & 1,9 & $-8,0$ \\
\hline
\end{tabular}

Fuente: Comisión Europea.

Para conseguir ese menor aumento se cuenta con una mayor generalización del gas natural en sustitución del carbón y de los productos petrolíferos, tanto en industria como en los sectores residencial y terciario, un incremento en el ahorro y eficiencia energética, una mayor penetración de las energías renovables y un alargamiento de vida en algunas centrales nucleares, como la de José Cabrera.

Las grandes ciudades españolas tienen todavia graves problemas de contaminación debido a la combustión masiva de energias altamente contaminantes. Eso se hace especialmente acuciante en momentos de estabilidad atmosférica, en los que la renovación del aíre es mínima. Es un problema general a todas las grandes aglomeraciones urbanas y se deriva del uso particular del automóvil, las calefacciones, la industria... La falta de un urbanismo adecuado, que haya sabido disociar las concentraciones industriales más agresivas de los espacios habitados, ha acuciado el problema. Las renovables lo reducirian, con un uso mayor del que tienen en la actualidad. Además, España ofrece grandes posibilidades al respecto, sobre todo en lo que se refiere a energía solar. Ocurre lo mismo 
con la minihidráulica. Los recursos hidráulicos españoles son importantes y están bastante utilizados, por lo que la nueva construcción de grandes embalses no es fácil y generan una fuerte oposición. Un ejemplo fueron las movilizaciones sociales en Riaño (León) y, más recientemente, en Itoiz (Navarra) y Santaliestra (Huesca).

En Septiembre de 1999 se sucedieron varias manifestaciones en la localidad pirenaica de Santaliestra, en la provincia de Huesca, para protestar contra el inicio de las obras de un embalse. La Confederación Hidrográfica del Ebro ( $\mathrm{CHE}$ ) tiene expropiadas unas 300 hectáreas en la zona de construcción del embalse, acogiéndose a una ley de 1954, ante la que no caben recursos. Las protestas se basan en el miedo de los vecinos del pueblo a la presa. Al parecer, y según diversos informes científicos, las laderas del vaso del futuro embalse presentan una elevada inestabilidad, por lo que podría haber importantes corrimientos de tierra. Esto podría poner en peligro la obra y causar daños a las poblaciones situadas aguas abajo del río Esera, como Santaliestra, a sólo 500 metros. Esta manifestación contó con el apoyo de Greenpeace y Ecologistas en Acción, opuestos también a otros embalses proyectados en la región, como el de Jánovas, Biscarrués, Yesa y Echago.

Por las mismas fechas, unas 1.500 personas se manifestaron en Guadalajara para protestar contra el proyecto de construcción de un almacén de residuos radiactivos en la central nuclear de Trillo y para pedir el cierre de la de Zorita, calificada de "obsoleta" por diversos grupos ecologistas.

Estas protestas, y otras más, demuestran la creciente conciencia ecológica que despierta la energía y su relación con la sociedad y el territorio. La mayor sensibilidad medioambiental en España moviliza cada vez a un número más importante de personas que rechazan cualquier proyecto energético que altere económica, social o ambientalmente una determinada zona.

\section{BIBLIOGRAFIA}

ALCAIDE,J. (1989) : "El sector gas natural: evolución y desarrollo futuro ante el Mercado Unico Europeo", Información Comercial Española ,670-671.

BADOSA PAGES, J. (1986) : "Gas natural en España: la energía de los años 90"

CAMPO AGUILERA, A. (1980): "La energía hidráulica en España. Situación actual y perspectivas", Boletín Informativo de la Fundación J. March, 90.

DIAZ-CANEJA BURGALETA, F. (1986): "El potencial hidroeléctrico de España", Papeles de Economía Española, 29. 
GARCIA ALONSO, J.M. e IRANZO MARTIN, J.E. (1989): La energía en la economía mundial y en España, Madrid, Editorial AC.

INSTITUTO PARA LA DIVERSIFICACIÓN Y AHORRO DE LA ENERGIA (1999): Las energias renovables en España. Balance y perspectivas 2000, Madrid.

IRANZO MARTIN, J.E. (1984): "Presente y futuro del sector energético español", Papeles de Economía Española, 21.

IRANZO MARTIN, J.E. (1987): "La energía nuclear en España", Situación, 2.

IRANZO MARTIN, J.E. (1992): "El sector energético español", Papeles de Economía Española, 50.

JARABO FRIEDRICH, F. y otros (1988): El libro de las energías renovables, Madrid, S.A. de Publicaciones Técnicas, col. "Era Solar".

LUCENA BONNY, A. (1988): Energias alternativas y tradicionales. Sus problemas ambientales, Madrid, Talasa Ediciones.

MARIN QUEMADA, J.M. (1987): "Petróleo 1987: investigación, producción, refino y comercialización en España", Situación, 2.

MENENDEZ PEREZ, E. (1998): Las energías renovables. Un enfoque político-ecológico, Madrid. Los Libros de la Catarata.

MINISTERIO DE INDUSTRIA Y ENERGIA (1998): La energia en España, Madrid.

MOLINA IBAÑEZ, M. y CHICHARRO FERNANDEZ, E. (1990): Fuentes de energía y materias primas, Síntesis, col. "Geografía de España".

PARDO ABAD, C.J. (1993): Las fuentes de energía, Madrid, Sintesis, col. "Espacios y Sociedades".

SANTAMARÍA, J. (1988): El petróleo en España. Del monopolio a la libertad, Madrid, Espasa-Calpe.

UNESA (1988): La electricidad en España, Madrid.

\section{NOTAS}

Los balances de energía van expresados en una unidad común que es la tonelada equivalente de petróleo (tep). Una tep se define como $107 \mathrm{Kcal}$. La conversión de otras unidades a tep se hace atendiendo a los poderes calorificos de la energía considerada.

2 El mineral de uranio se extrae en minas a cielo abierto o subterráneas. Una vez extraido el mineral, ess llevado a una serie de molinos trituradores que lo pulverizan y lo convierten en arena fina. Posteriormente, se disuelve el uranio mediante diferentes soluciones químicas, resultando de ello una mezcla de óxidos con una fórmula química equivalente $\mathrm{U}_{3} \mathrm{O}_{8}$, que constituye la materia prima para todos los procesos que, con el tiempo, conducirán al núcleo de la central y a la reacción en cadena. Dicha mezcla contiene un $85 \%$ de uranio en peso. 